\title{
Plinko Poetry
}

Describe what you made. We made a Plinko Poetry machine, an interactive installation that allows users to create blackout poetry. A player walks up a set of stairs to the top of a screen. From there they release a ball down onto the screen. A poem is then generated from words selected in the ball's path.

\section{Briefly describe the process of how this was made?}

Plinko Poetry started as a class project a few years ago; we iterated that project for portability. However, when we were invited to SIGGRAPH 2016 we began to think about how we could impress people in such a big space. We decided to build a large-scale machine to give people an immersive experience.
Then we tried to build a machine with projection using PVC pipe as a frame to support the projection surface. In this way the machine can be both large and lightweight.

\section{What expertise (skills and competences) did it require?}

This project involved both programming and hardware making. On the software side, we used openFrameworks to track the ball's movement with a webcam, control text scrolling onscreen, and print out the generated poem on a small thermal printer. Most of the code was reused from the previous iteration; we only needed to adjust it to fit the current setup.

On the hardware side, it was a totally different story.
We had never built such a huge setup on our own so we carefully designed everything in 3D CAD software to make sure everything would fit.

Then we made every part and built the giant machine in one pass.

\section{What materials and tools did you use?}

We used PVC pipe as a structure because it's lightweight, strong, and inexpensive. We used a PVC pipe cutter to cut the pipe to the correct length. Then we used a sanding machine to finely adjust the length and shrink the ends of the pipe for easy assembly.

The framework was built with PVC pipes and wood strips. We drilled holes in the wood strips for installing the acrylic pegs and added a white LED inside each peg.
Meanwhile, we cut holes in the projection canvas and secured those holes with eyelets. Those eyelets were also big enough to fit the acrylic pegs. This made installing and taking down the canvas fairly easy.

\section{Did anything go wrong?}

We planned to upload poems to our server in real time and control the LEDs wirelessly. Unfortunately we underestimated the radio noise during the exhibition and couldn't get a reliable connection. But the machine still worked without an Internet connection and the LED controller could run its own pattern, so this problem didn't compromise people's experience.

What is the one thing about making this that you would

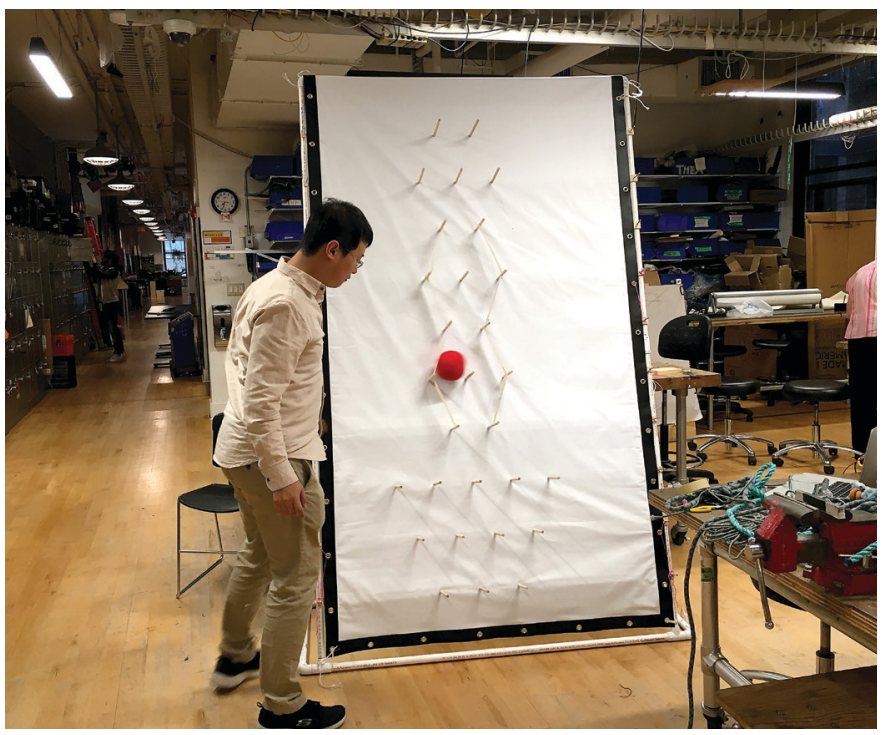

$\rightarrow$ Deqing testing a small-scale prototype.

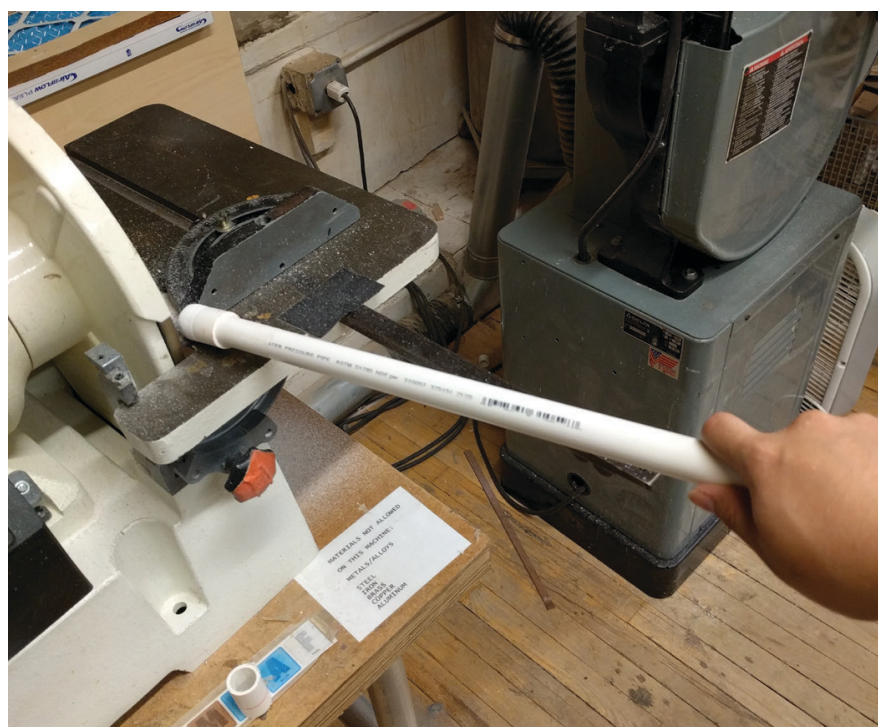

$\rightarrow$ Sanding the end of a tube. 
like to share with other makers?

PVC pipe is an ideal material for making big structures. During our making process, we realized there is a wide variety of pipe you can choose from, ranging from utility grade to furniture grade. Also, there is a wide variety of pre-made connectors to choose from. You can either fasten pipes with screws for a quick but not robust fix, or you can use glue to do it permanently.

(1) Deqing Sun, Thinkcreate LLC and NYU,

$\rightarrow$ dsathinkcreate.us

(1) Peiqi Su, Thinkcreate LLC and NYU,

$\rightarrow$ psQthinkcreate.us

(2) http://thinkcreate.us/plinko/

DOI: $10.1145 / 3065847$

COPYRIGHT HELD BY AUTHORS

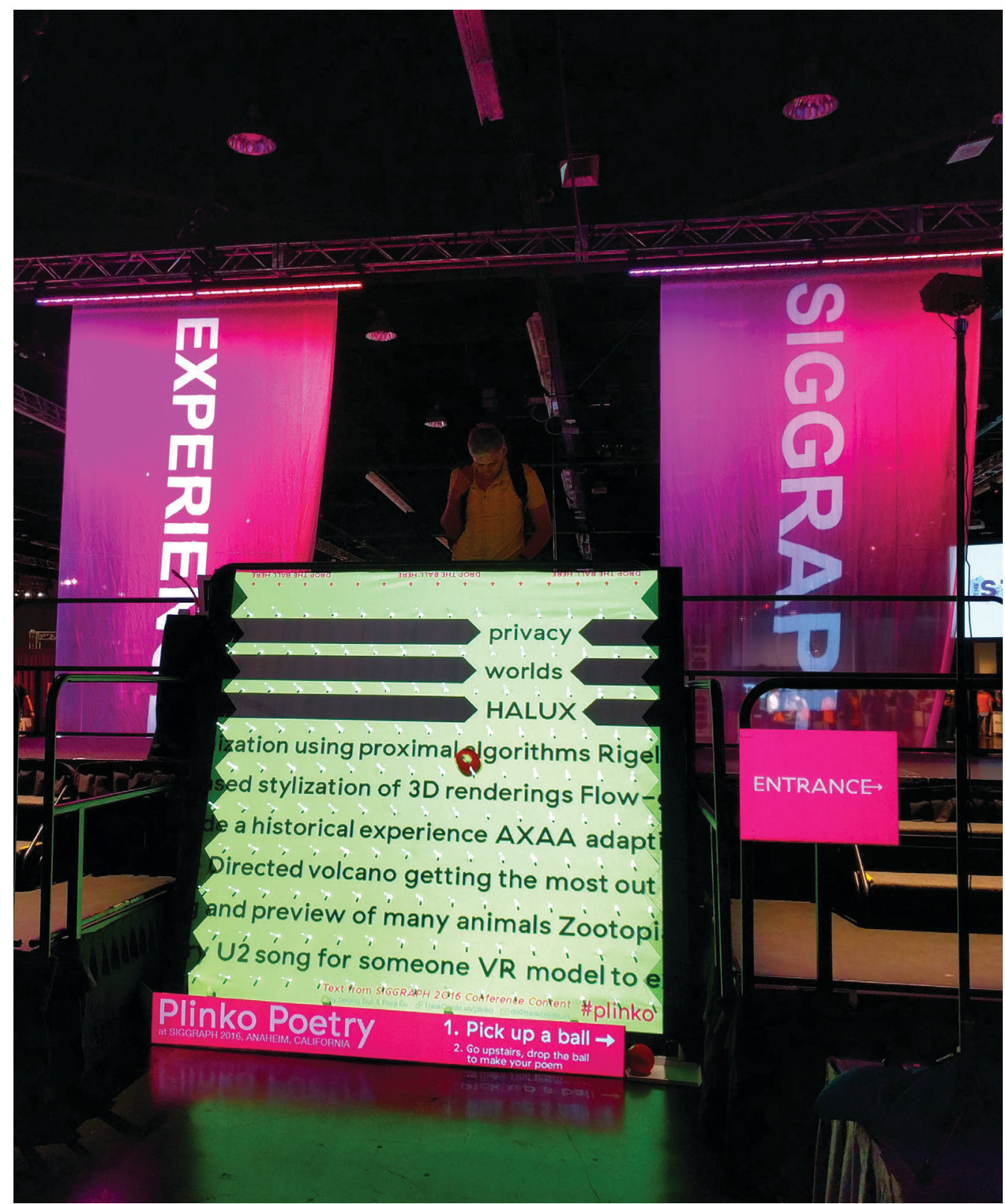

$\rightarrow$ Assembled Plinko Poetry at SIGGRAPH 2016.

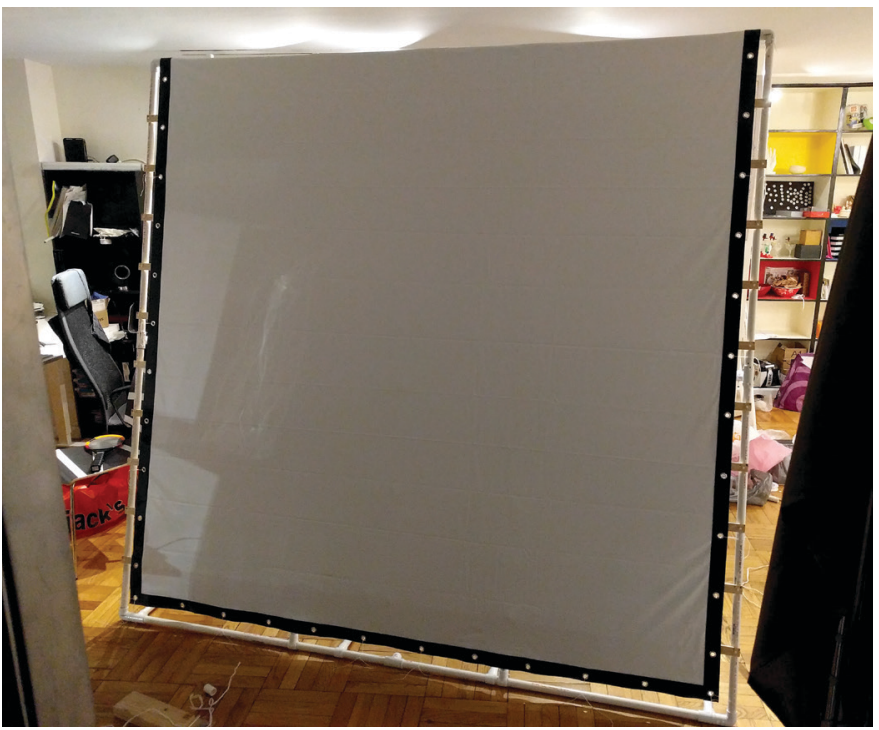

$\rightarrow$ Testing the frame at home.

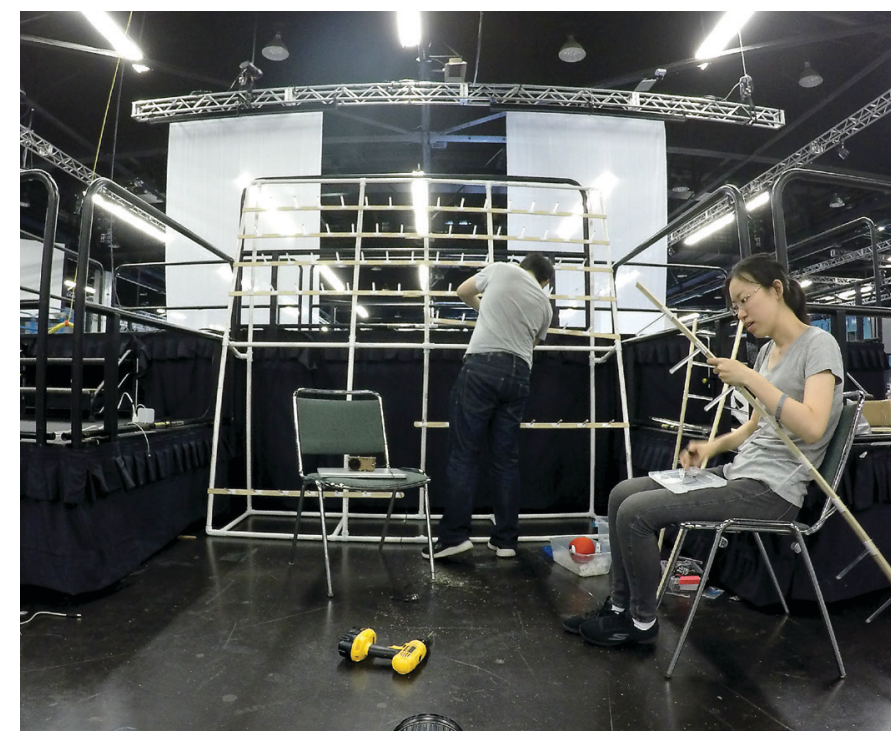

$\rightarrow$ Installing the frame at SIGGRAPH 2016. 